\title{
I QUADERNI DI EPOCA FASCISTA VEICOLO DI PROPAGANDA IDEOLOGICA E STRUMENTO DIDATTICO: IL FONDO DELLA SCUOLA ELEMENTARE PARINI DI TORINO (1938-1942)
}

\author{
Exercise books in the fascist era as a medium for ideological \\ propaganda and an educational tool: the case of the Parini \\ elementary school in Turin (1938-1942) \\ Los cuadernos de la época fascista vehículo de propaganda \\ ideológica e instrumento didáctico: el fondo de la escuela \\ elemental Parini de Turín (1938-1942)
}

\section{Maria Cristina Morandini ${ }^{\alpha}$}

Data di ricezione: 07/01/2019 • Data di accettazione: 04/02/2019

Riassunto. Nell'ambito della recente ricerca storico-educativa, non solo italiana, ha assunto una primaria importanza il filone dedicato alla «cultura materiale» della scuola, volto a ricostruire la vita reale e quotidiana all'interno delle aule. Tra le nuove tipologie di fonti utilizzate per documentare l'esperienza scolastica nella sua dimensione concreta figurano, accanto ai libri di testo, i quaderni. Presso la biblioteca del museo della scuola di Torino, sono conservati un' «artigianale» bibliotechina di classe e una serie di quaderni di italiano e di calligrafia, scritti dagli alunni delle classi quarta e quinta della locale scuola elementare Parini negli anni immediatamente precedenti la caduta del fascismo. Dall'analisi degli esercizi di «bella scrittura» e dalla lettura dei temi e dei resoconti relativi agli eventi di cronaca, nazionali e internazionali, appare evidente la massiccia propaganda ideologica del regime, finalizzata alla celebrazione, con toni retorici, della figura del Duce e della dittatura fascista con specifico riferimento agli orientamenti in politica interna ed estera e alla promozione di opere socio-assistenziali ed educative. Il materiale didattico esaminato offre inoltre interessanti spunti sull'attività didattica

\footnotetext{
${ }^{\alpha}$ Dpto. Filosofia e Scienze dell'Educazione, Università di Torino. V. Gaudenzio Ferrari, 9, 10124, Torino, Italia. maria.morandini@unito.it 
svolta in classe: dal programma di studi ai contenuti delle discipline; dalle modalità d'insegnamento e di correzione degli errori ai criteri di valutazione del profitto a breve e medio termine; dalla verifica dei progressi nella carriera scolastica alla percezione soggettiva del livello di conoscenze e dei margini personali di miglioramento.

Parole chiave: Quaderni; Calligrafia; Lingua italiana; Fascismo; Attività didattica.

Abstract. In recent historical-educational research, both in Italy and internationally, inquiry into the "material culture» of schools is seen as contributing crucially to the reconstruction of authentic everyday life in the classroom. The new sources used to document the specifics of educational experience include text books and exercise books. The library of Turin's school museum conserves a "homemade» class library and a set of Italian language and handwriting exercise books, the work of fourth and fifth grade students at the local Parini elementary school from the years immediately preceding the fall of the fascist regime. An analysis of the pupils' exercises in "good handwriting», essays, and summaries of Italian and international news events, brings to light the regime's large-scale ideological propaganda efforts, which are reflected in the use of rhetorical language celebrating the figure of Il Duce and the fascist dictatorship with specific reference to aspects of its domestic and foreign policy and social-welfare and education programmes. The material examined also offers interesting insights into the educational activities conducted in the classroom: from study plans to curricular contents; from teaching methods and the treatment of errors to short- and medium-term assessment criteria; from the formal testing of students' educational progress to subjective perceptions of their current knowledge and personal potential for improvement.

Keywords: Exercise books; Handwriting; Italian language; Fascism; Educational activities.

Resumen. En el contexto de la investigación histórica y educativa reciente, no solo en Italia, ha adquirido una importancia primordial la línea dedicada a la "cultura material» de la escuela, destinada a reconstruir la vida real y cotidiana dentro de las aulas. Entre los nuevos tipos de fuentes utilizadas para documentar la experiencia escolar en su dimensión concreta se incluyen, además de libros de texto, los cuadernos. En la biblioteca del museo de la escuela de Turín, una biblioteca artesanal de clase y una serie de cuadernos de caligrafía e italiano, escritos por los alumnos de cuarto y quinto grado de la escuela primaria local Parini en los años inmediatamente anteriores a la caida del fascismo. A partir del análisis de los ejercicios de "hermosa escritura» y de la lectura de los temas e informes relacionados con los eventos noticiables nacionales e internacionales, aparece la 
propaganda ideológica masiva del régimen, dirigida a la celebración, con tonos retóricos, de la figura del Duce y de la dictadura fascista con referencia específica a las orientaciones en política nacional y exterior y a la promoción de obras de bienestar social y educativas. El material educativo examinado también ofrece información interesante sobre la actividad didáctica llevada a cabo en el aula: desde el programa de estudio hasta los contenidos de las disciplinas; desde los métodos de enseñanza y corrección de errores hasta los criterios para evaluar el rendimiento a corto y medio plazo; desde la verificación del progreso en la carrera escolar hasta la percepción subjetiva del nivel de conocimiento y los márgenes pers onales de mejora.

Palabras claves: Cuadernos; Caligrafía; Lengua italiana; Actividad didáctica; Fascismo.

\section{UNO SGUARDO AL MATERIALE}

Nell'ambito della recente ricerca storico-educativa, non solo italiana, ha assunto una primaria importanza il filone dedicato alla «cultura materiale» della scuola, volto a ricostruire la vita reale e quotidiana all'interno delle aule, considerate come luogo di apprendimento e contesto di relazioni: è un'analisi che spazia dai contenuti disciplinari, alle metodologie, alle esperienze vissute dai singoli alunni e dal gruppo classe durante lo svolgimento delle lezioni e le uscite sul territorio. ${ }^{1}$ L'esigenza di documentare la realtà scolastica nella sua dimensione concreta ha indotto gli studiosi ad individuare nuove tipologie di fonti da affiancare a quelle tradizionalmente in uso: dai libri di testo ai quaderni; dagli arredi ai sussidi didattici; dai registri alle pagelle; dai diari scolastici alle memorie autobiografiche della classe docente.

I quaderni, nello specifico, sono stati oggetto in Italia di due importanti convegni che, promossi nel primo decennio del Duemila, hanno rappresentato un'occasione per un confronto con le ricerche già avviate

\footnotetext{
1 Tra gli studi più significativi si segnalano: Martin Lawn e Ian Grosvenor, Materialities of Schooling: Design, Technology, Objects, Routines (Oxford: Symposium Books, 2005); Agustín Benito Escolano (ed.), La cultura material de la escuola (Berlanga De Duero: CEINCE, 2007); Pierre Moegli, Les industries éducatives (Paris: Presses Universitaires de France, 2010); Juri Meda y Ana Badanelli (a cura di), La historia de la cultura escolar en Italia y en España: balance y perspectivas (Macerata: EUM, 2013); Simonetta Polenghi, «School subjects didactics in the history of education. Sources and methodology. Italian studies», History of Education \& Children's Literature 1 (2014): 635-648; Juri Meda, Mezzi di educazione di massa. Saggi di storia della cultura materiale della scuola tra XIX e XX secolo (Milano: Angeli, 2016).
} 
nel panorama internazionale: il primo su «I quaderni scolastici: una fonte per la storia dell'educazione», organizzato a Brescia il 27 ottobre 2005 dall'Università Cattolica del Sacro Cuore; il secondo dal titolo «School Exercise Books. A Complet Source for a History of the Approach to Schooling and Education in the 19th and 20th Centuries» che, tenutosi a Macerata dal 26 al 29 settembre 2007 grazie ad un'azione sinergica tra la locale sede dell'università e l'Istituto nazionale di documentazione per l'innovazione e la ricerca educativa (INDIRE), ha coinvolto 85 relatori provenienti da diverse parti del mondo. ${ }^{2}$

Dalla lettura dei contributi appare evidente la natura ibrida di una fonte che offre allo storico interessanti spunti per uno studio all'intreccio di una pluralità di ambiti disciplinari: dalla già richiamata storia della «cultura materiale» della scuola alla storia dell'illustrazione applicata agli oggetti didattici; dalla storia sociale della scrittura e dei processi di alfabetizzazione alla storia dei costumi educativi, alla storia dei processi economici connessi allo sviluppo della scolarizzazione. Se Juri Meda $^{3}$ e Anna Ascenzi ${ }^{4}$ hanno posto l'accento sulle complesse vicende relative alla produzione e commercializzazione in Italia dei quaderni di scuola nel rispetto della normativa vigente e in linea con le strategie politico-culturali del consenso adottate dallo Stato liberale e successivamente dalla dittatura fascista, Davide Montino ${ }^{5}$ si è soffermato sulle scritture e sui testi prodotti dall'alunno e veicolati dai quaderni di scuola

\footnotetext{
${ }^{2}$ Gli atti del convegno di Brescia sono stati pubblicati nella sezione monografica «I quaderni di scuola tra Otto e Novecento", Annali di Storia dell'Educazione e delle Istituzioni Scolastiche 13 (2006): 13-189, mentre quelli di Macerata sono confluiti nei due volumi, curati da Juri Meda, Davide Montino e Roberto Sani, School exercise books. A Complete Source for a History of the Approach to Schooling and Education in the 19 th and 20th Centuries (Macerata: Edizioni Polistampa, 2010). Sul dibattito storiografico avviato in questi anni in Italia attorno ai quaderni di scuola si veda il saggio di Roberto Sani, «Bilancio della ricerca sui quaderni scolastici in Italia», in Meda y Badanelli (a cura di), $\mathrm{La}$ historia de la cultura escolar en Italia y en España, 83-103.
}

3 Juri Meda, «Contro il tanto deprecato mercantilismo scolastico: i controversi rapporti tra produttori di quaderni, insegnanti e cartolai e l'intervento del regime fascista», in School exercise books, ed. Juri Meda et al. vol. I, 507-551.

${ }_{4}^{4}$ Anna Ascenzi, «Le cartiere Pigna e i quaderni scolastici della "Terza Italia” (1870-1945)», in School exercise books, ed. Juri Meda et al. vol. I, 487-505.

5 Davide Montino, «Da scolari a bambini? Scritture disciplinate e scritture personali nei quaderni di scuola», in School exercise books, vol. II, 1289-1303. Dello stesso autore si segnalano, inoltre, "Quaderni scolastici e costruzione dell'immaginario infantile», Annali di Storia dell'Educazione e delle Istituzioni Scolastiche 13 (2006): 167-189, e Bambini, penna e calamaio: esempi di scritture infantili e scolastiche in età contemporanea (Roma: Aracne, 2007). 
nell'intento di cogliere l'articolato ed eterogeneo immaginario infantile di cui sono espressione. Le relazioni di Gianluca Gabrielli, di Francesco Ascoli e di Alberto Barausse, dedicate, rispettivamente, ai quaderni di aritmetica, di calligrafia e di lingua e grammatica italiana mostrano, infine, il possibile utilizzo di questa fonte per uno studio sulle pratiche didattiche in ordine alle diverse discipline.

Alla luce di tali categorie interpretative ho analizzato una serie di quaderni conservati presso il Museo della Scuola e del Libro per l'Infanzia della Fondazione Tancredi di Barolo di Torino. ${ }^{6}$ Nello specifico, ho focalizzato l'attenzione sul quaderno secondo una duplice prospettiva: come veicolo di propaganda ideologica allo scopo di verificare, attraverso l'analisi delle «scritture scolastiche» e dei disegni ad esse correlati, la reale incidenza degli ideali del regime sulle coscienze e sul vissuto personale degli allievi; come strumento di attività didattica, non circoscritta ai contenuti e ai metodi d'insegnamento, ma indagata anche sotto il profilo dei criteri di correzione/valutazione adottati dal maestro e di quello della percezione soggettiva degli allievi in merito al profitto personale ed agli eventuali margini di miglioramento.

Il fondo è costituito da 33 quaderni (9 di calligrafia, 24 di italiano) scritti, negli anni immediatamente precedenti alla caduta del fascismo, da 12 alunni che frequentano la quarta e la quinta classe maschile della scuola elementare «Giuseppe Parini», ubicata in una zona vicino al centro del capoluogo piemontese. ${ }^{7}$ Per l'insegnamento della lingua vengono utilizzate due differenti forme di avviamento al comporre, distinte in altrettanti quaderni: da un lato il "diario», semplice resoconto di episodi giornalieri o di avvenimenti nazionali o internazionali, dall'altro lo

\footnotetext{
${ }^{6}$ I quaderni rientrano in un più ampio fondo che, catalogato all'interno della sezione «Materiali scolastici per la didattica», è costituto da circa 1.200 unità, risalenti al periodo compreso tra la seconda metà dell'Ottocento e la metà del Novecento. Sulla storia e sul ricco ed eterogeneo patrimonio librario della Fondazione si vedano Pompeo Vagliani, Francesca Pizzigoni, «La collezione di quaderni del Museo della scuola e del libro per l'infanzia di Torino", in School exercise books, vol. I, 115-126 e Fabiano Loparco, «The MUSLI (Museo della Scuola e del Libro per l'Infanzia) of the Foundation Tancredi di Barolo in Turin. An institution at the forefront of the preservation and enhancement of educational and scholastic heritage», History of Education \& Children's Literature 1 (2013): 795-818.

${ }_{7}$ L'intitolazione al poeta Giuseppe Parini (1729-1799) risale all'anno 1911. In precedenza la scuola, sorta nel 1882, prendeva il nome dalla borgata Aurora in cui, ancora oggi, è ubicata. A fine Ottocento la sede è protagonista di una serie di iniziative pionieristiche promosse, in materia d'istruzione, dall'amministrazione comunale: dall'istituzione del servizio di mensa scolastica all'apertura di ricreatori festivi in cui gli alunni hanno la possibilità di trascorrere il tempo libero.
} 
svolgimento di temi con un soggetto stabilito a priori dal maestro. ${ }^{8}$ Unico, invece, è il quaderno di bella scrittura in cui figurano esercizi di progressiva difficoltà con il passaggio dalla riproduzione delle lettere dell'alfabeto e dei numeri, alle parole, a brevi dettati. La copertina dei quaderni, originale o foderata, è monocromatica, adorna di un semplice adesivo su cui, accanto al nome, sono riprodotti i colori della bandiera italiana con i simboli patriottici e fascisti (figura 1). ${ }^{9}$

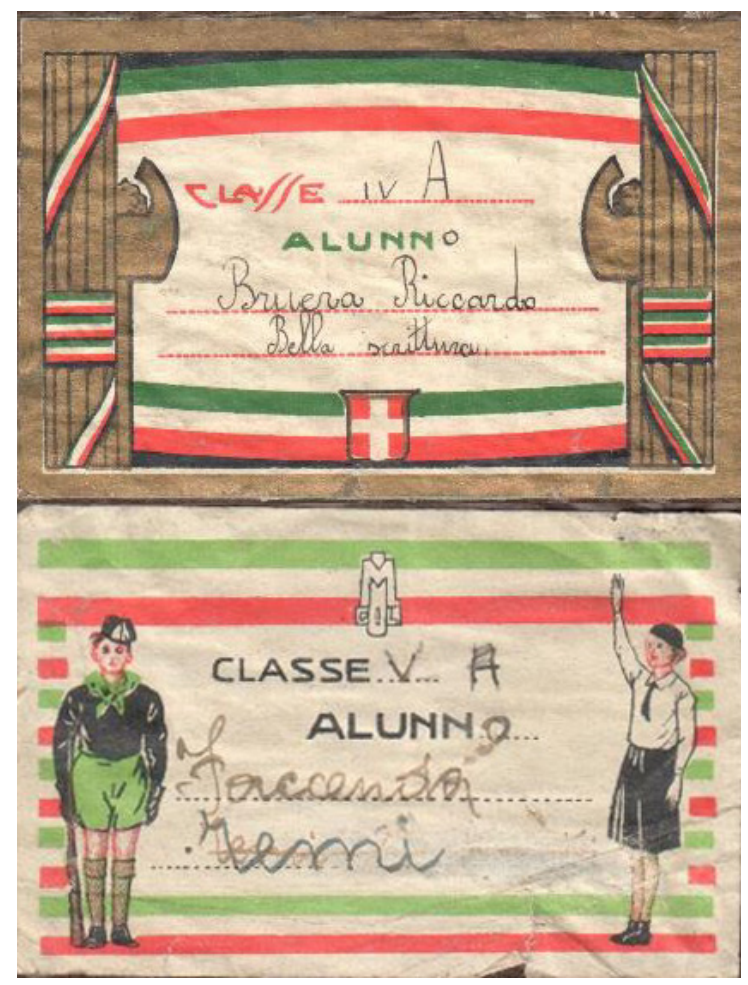

Figura 1. Etichette adesive sulle copertine dei quaderni.

\footnotetext{
8 È una distinzione prevista dalla normativa come si evince dall'elenco del «Materiale occorrente nelle varie classi per uso dello scolaro», pubblicato nei «Programmi di studio e prescrizioni didattiche per le scuole elementari» del $1^{\circ}$ ottobre del 1923 (Regio Decreto, n. 2185). Per il testo integrale del documento si veda Franco V. Lombardi, I programmi per la scuola elementare dal 1860 al 1985 (Brescia: La Scuola, 1987), 361-365.

${ }_{9}$ Su alcuni è ancora visibile il contrassegno che cartolai e librai sono tenuti ad applicare per legge, a partire dal 1931, sui quaderni in vendita al pubblico: acquistato dalle Confederazioni Provinciali del Commercio, si configura come una sorta di contributo fiscale indiretto a sostegno delle attività dei patronati scolastici rivolte agli alunni delle famiglie povere. Sull'argomento si veda Meda, Mezzi di comunicazione di massa, 80-124.
} 
La prima pagina, una sorte di frontespizio in cui compaiono informazioni relative all'identità dell'alunno, alla classe frequentata, all'anno scolastico, alla disciplina e al nome dell'insegnante, è decorata con una greca o arricchita da uno o più disegni a soggetto libero. Ogni esercizio di bella scrittura, cronaca di un avvenimento o tema è seguito da una illustrazione al fondo della pagina o negli appositi fogli bianchi predisposti a lato. La tipologia delle righe differisce in relazione alla disciplina o alla classe: la spaziatura all'interno della quale l'alunno scrive, sempre in carattere corsivo, è più stretta nei quaderni di calligrafia e in quelli di lingua di classe quarta, più ampia nella raccolta dei componimenti illustrati e nei diari dell'ultimo anno del ciclo elementare. È una scelta che si spiega da un lato con le esigenze e le peculiarità della singola materia, dall'altro con la consapevolezza della progressiva acquisizione, da parte dell'allievo, di sicurezza e padronanza stilistica nella scrittura.

All'interno del fondo è stato possibile reperire anche un originale e curioso materiale didattico, rappresentato da una bibliotechina «artigianale» prodotta dagli alunni: si tratta di una trentina di opuscoli, di piccolo formato, funzionali alla celebrazione del regime e all'approfondimento delle tematiche storico-geografiche o scientifiche affrontate durante le lezioni. Sulla copertina, in cartoncino a tinta unita, compaiono il numero della serie, il titolo in stampatello maiuscolo e, talvolta, il nome dell'autore di cui si ripropone il testo. L'interno è costituito da circa una decina di fogli bianchi in cui la parte scritta, costituita da ritagli stampati disposti in colonne, si alterna a immagini in bianco e nero: in alcuni casi sono presenti anche disegni dell'allievo che danno un tocco personale al libretto. ${ }^{10}$

\section{IL QUADERNO COME VEICOLO DI PROPAGANDA IDEOLOGICA}

Negli esercizi di calligrafia e nei testi di lingua ricorre, in svariate forme e con toni retorici, la celebrazione del regime: dal richiamo agli eventi relativi alle origini e alla storia della dittatura fascista alla costruzione del mito attorno alla figura del Duce; dal plauso per le scelte di

\footnotetext{
${ }_{10}$ Un'eccezione è rappresentata dagli opuscoli relativi ad argomenti di carattere geografico («Il Po», «Il Piemonte», «La Toscana»). In questi casi si tratta dell'esito di una ricerca, trascritta dallo stesso allievo e corredata da cartine geografiche e immagini a colori o in bianco e nero. Nell'ultima pagina compare la firma del maestro con l'indicazione della data di conclusione del lavoro.
} 
politica interna all'orgoglio per la costituzione dell'impero, emblema di una nazione forte e faro di civiltà nel mondo; dall'esaltazione del mito della romanità all'elogio delle opere socio-assistenziali ed educative. Nei quaderni di «bella scrittura» gli alunni sono invitati a ricordare l'anniversario della fondazione dei fasci di combattimento (23 marzo 1919) e la salita al potere di Mussolini con la marcia su Roma (28 ottobre 1922) oltre a riprodurre motti ( Credere, obbedire, combattere, vincere») e frasi, pronunciate dal capo di governo, in cui si inneggia all'importanza del lavoro quotidiano, "oscuro», umile e silenzioso come alla più alta $\mathrm{e}$ lodevole forma di servizio alla patria. Il capo delle Camicie Nere rappresenta a tale riguardo un fulgido esempio: «Mi vanto — si legge in una sua frase dettata dal maestro- di essere figlio di lavoratori. Mi vanto di aver lavorato con le mie braccia. Ho conosciuto le umili fatiche della gente che lavora». ${ }^{11}$

Nella sintesi dei discorsi di Mussolini, riproposti nella lettura della stretta attualità, viene tratteggiata l'immagine di un uomo di Stato espressione della gente comune e, al tempo stesso, dalla personalità eccezionale: se da un lato si usa l'appellativo «il nostro amato Duce», che rivela un intenso coinvolgimento emotivo e una chiara percezione della sua vicinanza al popolo, dall'altro se ne enfatizza il carattere straordinario, evidente nella scelta di usare l'iniziale maiuscola negli aggettivi possessivi e nei pronomi («Lui», «Egli») che a lui si riferiscono. Si tratta di una figura carismatica, capace di parlare al cuore e alle coscienze e di infondere negli animi la certezza dell'imminente esito positivo della guerra: «Dopo la Sua parola — scrive l'alunno Chiavassa- nessuno più in Italia e nel mondo intero ha il minimo dubbio e tutti attendono ormai il momento vicino di salutare vittoriosi e carichi di gloria i nostri prodi combattenti».12

\footnotetext{
${ }_{11}$ Sergio Bergamasco, Quaderno di calligrafia, Classe V, anno 1941-1942. Il lavoro è il soggetto dei temi assegnati, nel marzo 1941, in classe quinta: l'alunno è invitato a descrivere le diverse tipologie dell'attività intellettuale e manuale in relazione ai contesti in cui essa abitualmente si svolge. Non manca il riferimento a quella scolastica che coinvolge insegnanti ed allievi: i primi veicolano le conoscenze ed educano alla disciplina; i secondi devono «stare attenti alle spiegazioni dei loro maestri ed eseguire con cura i loro compiti» (Riccardo Bruera, Lavoro intellettuale, lavoro meccanico, lavoro manuale, lavoro scolastico, Temi, Classe V, 1941-1942).
}

12 Gabriele Chiavassa, Discorso del Duce, Diario, Classe IV, anno 1940-1941. 
È il compimento della promessa del regime di restituire alla patria le gloriose vestigie del passato. Il recupero della memoria dell'antica Roma, caput mundi, passa attraverso la celebrazione dei soldati, di ogni ordine e grado, di cui non si conosce l'identità: il maestro dedica una lezione al poema di Virgilio, che narra le avventure di Enea, capostipite dei fondatori della città eterna, ${ }^{13}$ ma non disdegna di commemorare, nel dettato di «bella scrittura», il semplice legionario («Qui giace un soldato romano fermati viandante e inchinati riverente: dove giace un soldato romano è Roma»). Le origini della capitale italiana con specifico riferimento alla data di fondazione (il XXV aprile) e le note vicende di alcuni consoli della repubblica romana (Attilio Regolo, Scipione l'Africano) sono ricostruite negli opuscoli della già ricordata «Bibliotechina» di classe. Costante è anche la presenza dei simboli identificativi di quest'epoca storica: è sufficiente esaminare i disegni colorati con cui gli alunni abbelliscono le prime pagine dei quaderni per scorgere l'immancabile raffigurazione del fascio littorio (figura 2).
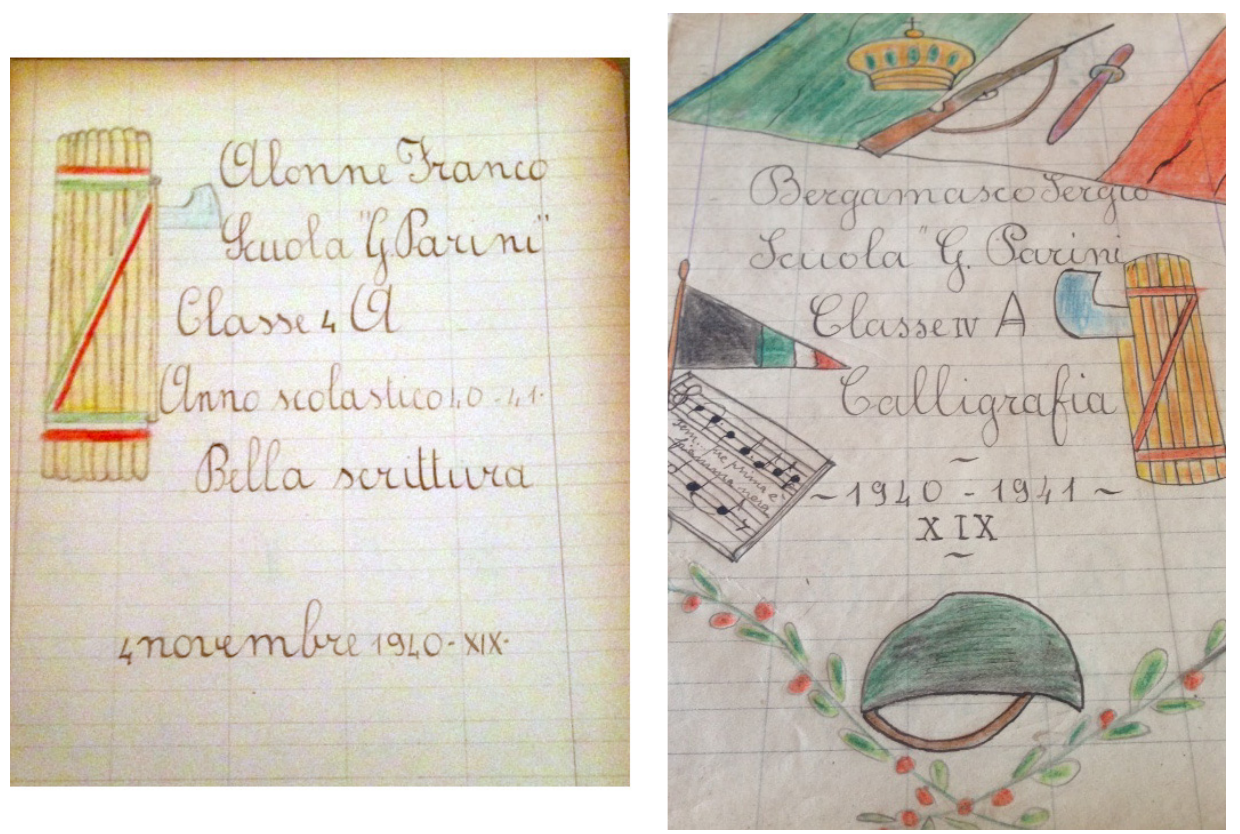

Figura 2. Frontespizi di quaderni decorati dagli alunni.

\footnotetext{
13 All'eroe troiano è dedicato anche l'opuscolo numero 5 della «Bibliotechina» di classe dal titolo $\mathrm{La}$ leggenda del mare italico.
} 
Una particolare attenzione è rivolta anche alle vicende belliche del 19151918 che, per l'Italia, coincidono con l'atto conclusivo del processo di unificazione della penisola. ${ }^{14}$ Emblematica al riguardo è la scelta di dedicare uno degli opuscoli della «Bibliotechina» all'eroico affondamento della corazzata navale austroungarica «Wien», avvenuto il 10 dicembre 1917 nelle acque di Trieste. Il protagonista dell'impresa è l'ammiraglio Luigi Rizzo (1887-1951), figura che incarna quella «ideale» continuità, più volte sottolineata dal regime, tra l'epopea della «Grande guerra» e la dittatura fascista: egli, infatti, prende parte nel 1935 alla campagna d'Etiopia, che pone le basi per la costituzione dell'impero, e, cinque anni dopo, alle prime fasi del secondo conflitto mondiale. ${ }^{15}$

Nei temi, in modo particolare, è posto l'accento sui brillanti risultati del regime nell'azione di contrasto alle sanzioni economiche e nella positiva risoluzione del contenzioso, in atto dal 1870, tra lo Stato italiano e la Chiesa cattolica. L'obiettivo della "vile Inghilterra di affamarci e di umiliarci», si legge nei quaderni, è reso vano dalla politica autarchica del Duce, basata sulla volontà di soddisfare il fabbisogno economico della nazione, facendo leva, unicamente, sulla produzione interna grazie ad un'opera di bonifica delle zone paludose e allo sfruttamento intensivo della terra. ${ }^{16} \mathrm{La}$ "battaglia del grano» è più volte menzionata nei componimenti dedicati alla coltivazione dei campi, all'utilità delle piante e al processo di lavorazione del pane. Così si esprime un alunno:

Come nella guerra reale contro il nemico vengono premiati i soldati più coraggiosi e che meglio si distinguono, così —si legge nel testo— nella «Battaglia del Grano» vengono premiati dal Duce in persona, con diplomi, medaglie e grossi premi in denaro, i contadini che con maggior lavoro, maggiori cure e miglior intelligenza hanno migliorato ed aumentato la produzione del grano. ${ }^{17}$

\footnotetext{
${ }_{14} \mathrm{Al}$ termine della «Grande guerra» vengono infatti annessi al Regno d'Italia il Trentino Alto Adige oltre alle province di Trieste e Gorizia e alla penisola istriana. Quest'ultima dopo il secondo conflitto mondiale fu assegnata alla Jugoslavia.

${ }^{15}$ L'ammiraglio siciliano si distingue, nelle missioni di guerra, per audacia, visione strategica e doti di comando, premiate con il conferimento di due medaglie d'oro e di quattro d'argento al valore militare. Per ulteriori approfondimenti si veda Fabio Andriola, Luigi Rizzo (Roma: Ufficio storico della marina militare, 2000).

${ }^{16}$ La logica autarchica è evidente, a livello culturale, nella volontà di tradurre il vocabolo straniero di uso corrente con quello equivalente in lingua italiana. Non a caso in uno dei titoli dei temi assegnati in classe quarta, nel gennaio del 1940, compare l'espressione «diporti» in sostituzione del termine «sport».

${ }_{17}$ Gabriele Chiavassa, I lavori del contadino, Temi, Classe IV, anno 1940-1941.
} 
Non manca la celebrazione dell'anniversario della firma dei Patti Lateranensi (11 febbraio 1929), atto destinato a «restituire pienamente l'Italia a Dio e Dio all'Italia». ${ }^{18}$ Si sottolinea con enfasi il merito del fascismo nel ricomporre la «dolorosa» e innaturale frattura tra il Papato e le istituzioni politiche di un paese profondamente cattolico, per storia e tradizione: da quella data i due stati sovrani, l'Italia e il Vaticano, riconosciutisi reciprocamente, vivono «in regolare accordo» e in spirito di amicizia. Come ricorda l'alunno Rosina, ricompaiono i simboli religiosi nei luoghi pubblici («Si fece mettere in ogni parte il crocifisso»), indice di un atteggiamento di venerazione e di rispetto che si esprime anche nella condanna del linguaggio blasfemo ( Quello che bestemmia viene punito»).

Alla luce dell'avvenuta conciliazione non sorprende l'ampio spazio attribuito alla dimensione religiosa nella scelta degli argomenti dei temi: basti pensare che nell'anno scolastico 1940-41 tutti i componimenti mensili di dicembre, assegnati in classe quarta, hanno come soggetto il Natale e quelli di aprile la Pasqua. ${ }^{19}$ Alle principali feste del calendario liturgico si affiancano le biografie dei santi e la memoria dei luoghi di culto con un particolare riferimento alla realtà locale: dal ricordo di San Giovanni Bosco, ${ }^{20}$ alla ricostruzione della storia della Sacra Sindone, conservata nel Duomo di Torino, alla descrizione della «grandiosa e monumentale» basilica di Superga, sacrario, nei sotterranei, di Casa Savoia.

Dell'introduzione delle leggi razziali italiane, nel 1938, non vi è traccia nei quaderni..$^{21}$ Diverso è il discorso per quanto concerne la «Bibliotechina» di classe: tra gli opuscoli ne figura infatti uno dal significativo titolo

\footnotetext{
18 Marco Velatta, La Conciliazione, Diario, Classe IV, anno 1940-1941.

${ }^{19}$ I titoli dettati dal maestro nel mese di dicembre sono i seguenti: $1^{\circ}$ La nascita di Gesù; $2^{\circ}$ Il Natale si avvicina: fervono i preparativi in casa e fuori; $3^{\circ}$ Come io mi preparo a festeggiare il Santo Natale; $4^{\circ}$ Le cerimonie della Chiesa: i doni di Natale. Quattro sono anche i temi relativi alla Pasqua: $1^{\circ}$ Gesù entra in Gerusalemme; $2^{\circ}$ L'ultima cena; $3^{\circ}$ Passione e morte di Gesù; $4^{\circ}$ La Resurrezione.
}

${ }^{20}$ La sua figura è oggetto di strumentalizzazione politica da parte del regime in un periodo in cui i rapporti tra il fascismo e la chiesa sono particolarmente tesi: nella commemorazione, tenuta a Roma nel 1934, il ministro dell'Educazione Nazionale definisce don Bosco, canonizzato quello stesso anno, come «il più italiano dei Santi» e un esempio di "perfezione italiana» che, diffuso nel mondo, incarna il mito della romanità. Per ulteriori approfondimenti si rimanda a Maria Cristina Morandini, «La figura di don Bosco nella politica scolastica del regime fascista: uno sguardo ai manuali di pedagogia», in Percezione della figura di don Bosco all'esterno dell'Opera Salesiana (eds.), Grazia Loparco e Stanislaw Zimniak (Roma: Las, 2016), 125-126.

${ }^{21}$ Le leggi razziali, promulgate in Italia tra il 5 settembre e il 17 novembre del 1938, decretano l'esclusione degli ebrei dalla vita sociale, dal mondo lavorativo, dall'insegnamento e dalle scuole 
L'impresa di Ario. Il testo, ricco di immagini in bianco e nero, si differenzia dagli altri anche per una maggiore lunghezza: è costituito da ben 28 pagine a fronte di una media che si attesta sulla decina. In esso si racconta la storia di un popolo di nomadi che, sotto la guida di un saggio anziano di nome Ario, decide di abbandonare la propria terra, funestata dalla carestia, per raggiungere, attraverso un viaggio faticoso e ricco di incognite, le alte e nevose montagne, visibili in lontananza. Nel corso della narrazione si colgono riferimenti alla teoria della razza ariana, identificabile con precise caratteristiche fisiche e temperamentali, ritenuta superiore alle altre e, in quanto tale, destinata, quasi per volontà divina, «al dominio del mondo». Fin dalle prime righe, infatti, si parla di gente "gagliarda e vigorosa» con «chiome bionde e brune» che fluiscono «su larghe spalle, incorniciando visi bianchi dai nobili lineamenti, dagli occhi belli ed intelligenti, dal mento forte e volitivo»; gente «superbamente virile», composta da «uomini atletici, insuperabilmente veloci e resistenti» (figura 3).

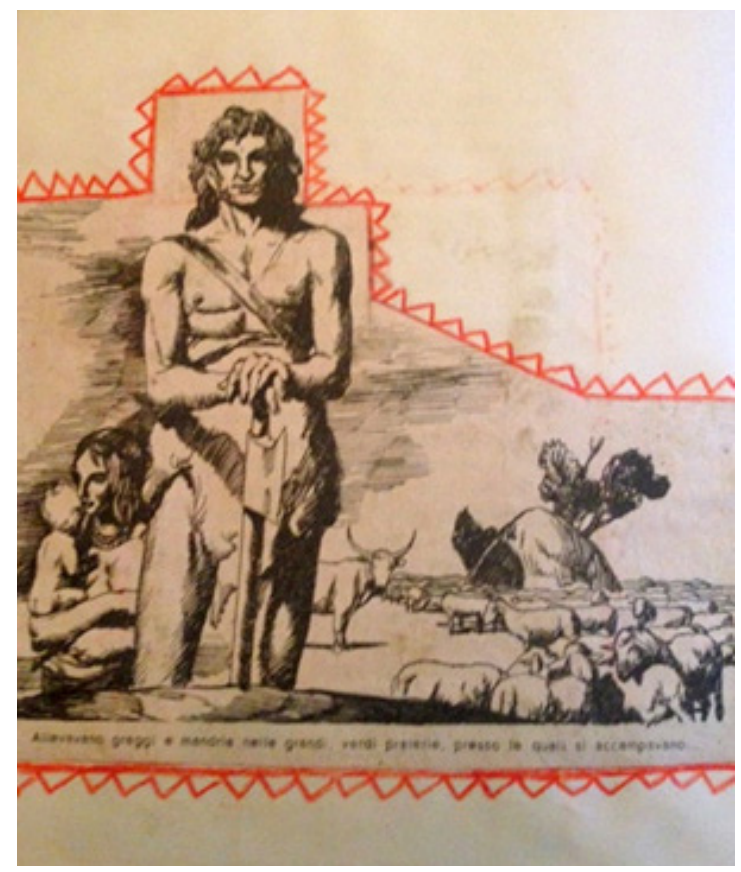

Figura 3. Raffigurazione della prestanza fisica e virile del popolo ariano.

pubbliche. Sulla campagna antisemita promossa dal regime si veda Michele Sarfatti, Gli ebrei nell'Italia fascista. Vicende, identità, persecuzione (Torino: Einaudi, 2007). 
Sono qualità che appaiono ancora più evidenti nel confronto impari con i Mongoli, definiti, con, un'accezione negativa, «gialli», «selvaggi» e non all'altezza dei «bianchi», per agilità, forza ed intelligenza: non a caso vengono ripetutamente sconfitti dai nemici e costretti a scendere a patti con loro. Non meno significativo appare, in conclusione, l'invito rivolto da Ario, in punto di morte, ai compagni di avventura, una sorta di testamento spirituale in cui esplicita è l'esortazione ad evitare ogni forma di contaminazione tra le razze: «Mantenete puro il vostro sangue ed il mondo sarà per voi».

Ricorrente, invece, è il tema della guerra come eco delle battaglie e come realtà destinata ad incidere nella vita quotidiana. ${ }^{22}$ Non manca il puntuale ed enfatico resoconto degli avanzamenti delle truppe italiane, impegnate, su più fronti, in azioni terrestri, navali ed aeree: dalla Grecia al Dodecaneso; dalla Libia all'Etiopia; dal Mediterraneo alla Russia. Le sconfitte maturano sempre al termine di un'eroica e strenua resistenza da parte di armate in netta inferiorità numerica e meno equipaggiate di quelle nemiche. Traspare con chiarezza, dagli elaborati, la convinzione della necessità del conflitto, combattuto a nome e in difesa non solo degli interessi nazionali: l'evento bellico è, infatti, descritto da Chiavassa come unica possibilità di liberazione, anche per «i popoli minori imploranti ed oppressi», dalla forma di schiavitù «secolare e odiosa», esercitata dall'Inghilterra «mediante la spadroneggianza dei mari e col possesso di tutti i passaggi obbligati e della maggior parte delle colonie nel mondo». ${ }^{23}$ In questa prospettiva acquistano un preciso significato i sacrifici della gente comune, chiamata a condividere, con i soldati, il peso e le sorti del conflitto: dal razionamento dei generi alimentari al riciclo degli abiti; dall'economia di luce e di energia elettrica all'uso limitato dei combustili.

\footnotetext{
${ }^{22}$ L'Italia entra in guerra il 10 giugno del 1940 a fianco di Germania e Giappone. L'8 settembre del 1943, il governo del generale Badoglio formatosi dopo la destituzione di Mussolini, avvenuta nel luglio di quello stesso anno, firma l'armistizio con gli Alleati. La liberazione dalle forze nazifasciste, il 25 aprile 1945, è possibile grazie all'intervento delle truppe americane che, sbarcate in Sicilia, risalgono la penisola e alla resistenza partigiana nelle regioni settentrionali.

${ }^{23}$ Gabriele Chiavassa, Il discorso del Duce, Diario, Classe IV, anno 1940-1941. Un concetto analogo è espresso, dallo stesso alunno, in un saggio di classe quinta: «La Vittoria sarà premio e gioia per noi tutti, tranquillità e benessere per tutti i popoli che, per questa guerra, saranno redenti» (Primo gennaio 1942, Diario, Classe V, anno 1941-1942).
} 
Sono privazioni destinate a modificare le abitudini degli italiani sui luoghi di lavoro (introduzione dell'orario unico negli uffici), in aula (riduzione di un mese del calendario scolastico) e in chiesa (anticipazione al pomeriggio della messa di Mezzanotte della vigilia di Natale). In alcuni casi si tratta di scelte volontarie del singolo che attinge ai risparmi personali ( La sottoscrizione spontanea della Nazione ai buoni del tesoro è veramente grande, sorpassa i 18 miliardi») o dona oggetti dal profondo valore affettivo ( «Le donne italiane, prima fra tutte la Regina Elena, offrono la fede d'oro alla Patria»). Sono sollecitati a dare il proprio contributo anche gli allievi che, grazie a piccole rinunce, si procurano il denaro per l'invio al fronte, durante le festività, di pacchi con vestiti, dolci e sigarette: è un semplice gesto che si affianca al ricordo quotidiano nelle preghiere serali. La popolazione convive con il sentimento della paura, originato dal pericolo di bombardamenti. Carlo Righetti, nel Diario di classe quarta, parla del suono delle sirene d'allarme per le incursioni dell'aviazione inglese sulla città di Torino e della conseguente necessità di trovare riparo nei rifugi antiaerei o in luoghi di fortuna. $^{24}$

Numerosi sono anche i riferimenti alle opere, promosse dal regime, nei diversi ambiti della società civile, a sostegno non solo delle classi povere: dalle iniziative assistenziali in favore dei lavoratori (ambulatori, le casse mutue) e delle mamme e dei neonati (Opera Nazionale Maternità ed Infanzia, asili) all'istituzione della Milizia Volontaria della Sicurezza Nazionale (MVSN) che, comunemente nota con il generico appellativo di Camicie Nere, è deputata al mantenimento dell'ordine pubblico e alla difesa della patria in epoca di pace e di guerra; 25 dai circoli ricreativi come il dopolavoro con la proposta del gioco delle bocce e l'offerta di

\footnotetext{
${ }^{24}$ Numerosi sono i bombardamenti del capoluogo piemontese tra il giugno del 1940 e il novembre 1943. Durante quello del 13 luglio 1943 è colpita anche la scuola Parini: 6 sono le aule distrutte, 23 quelle danneggiate. Le lezioni riprendono, nelle settimane successive, in uno stabile privato, preso in affitto, in via La Salle.

${ }^{25}$ Istituita il 23 gennaio 1923, sulla base del reclutamento volontario, comprende: la milizia ordinaria che organizzata in legioni, coorti e centurie, secondo lo schema dell'esercito dell'antica Roma, ha carattere territoriale; le milizie speciali suddivise in ferroviaria, forestale, portuale, postelegrafonica e stradale. Numerosi contingenti partecipano alla campagna italo-abissina e alla seconda guerra mondiale, segnalandosi per atti di eroismo, destinati a rimanere impressi nella memoria degli alunni: è il caso, ad esempio, di Bruera che riporta il numero esatto degli uomini della milizia decorati con medaglie (815) o con croci di guerra (1692) e caduti (3248) o feriti (6515) sui campi di battaglia.
} 
spettacoli cinematografici alle associazioni giovanili come l'Opera Nazionale Balilla (ONB) e la Gioventù Italiana del Littorio (GIL) che, oltre a gestire il tempo libero dei soggetti di età compresa tra i 6 e i 21 anni (emblematica è la festa della Befana fascista), organizzano campeggi e soggiorni in colonie montane e marine in cui «i fanciulli d'Italia possono crescere forti, sani e robusti». ${ }^{26}$ Il termine dialettale genovese «Balilla», che richiama alla memoria il ruolo svolto dal giovane Battista Perasso nella liberazione del capoluogo ligure dalla dominazione asburgica nel 1746, è scelto dal Duce come «soprannome» per le nuove generazioni, chiamate, sul suo esempio, a dare prova dell'orgoglio e del coraggio necessari per essere degni di una grande patria. Il processo di immedesimazione nel coetaneo eroe settecentesco è evidente nelle pagine in cui gli alunni sono invitati dal maestro a fornire una descrizione fisica e morale di sé stessi. È ancora una volta Gabriele Chiavassa a sintetizzare, in maniera efficace, il pensiero anche dei meno brillanti compagni di classe:

Sono italiano —egli scrive- e ne sono superbo; sono Balilla anziano e anche di questo me ne vanto; a suo tempo spero di essere un buon soldato [...]. Sono italiano e fascista e benché piccolo sento e cerco di capire la grande guerra che noi combattiamo, seguo con orgoglio le prodezze e gli eroismi dei nostri aviatori, marinai e soldati. ${ }^{27}$

Il testo relativo al profilo personale è affiancato da disegni altrettanto rivelatori di una radicata adesione ai modelli educativi veicolati dal fascismo: non mancano, infatti, allievi che si rappresentano con la divisa da balilla (figura 4). In alcuni casi il processo di identificazione è tale da indurre il maestro a scrivere al termine dell'elaborato: «È consigliabile

\footnotetext{
${ }^{26}$ Riccardo Bruera, Le opere del regime fascista, Diario, Classe IV, anno 1940-1941. L'opera Nazionale Balilla, fondata nel 1926, è un'organizzazione del regime volta a favorire il controllo del fascismo sulla formazione extrascolastica delle nuove generazioni. Inquadra tutta la gioventù italiana tra sei e diciotto anni, suddividendola per fasce d'età in ordine al sesso di appartenenza: le sezioni dei Figli della Lupa, dei Balilla e degli Avanguardisti per i maschi; quelle delle Figlie della Lupa, delle Piccole e delle Giovane Italiane riservate alle femmine. Nel 1937 l'O.N.B. viene trasformata nella Gioventù italiana del Littorio in cui confluisce anche la Federazione dei fasci giovanili di combattimento che raggruppa i soggetti dai diciotto ai ventuno anni.

${ }_{27}$ Gabriele Chiavassa, Il mio ritratto fisico e morale, Temi, Classe IV, anno 1940-41. Simili sono le parole utilizzate da Angelo Dotto nella presentazione di sé stesso: «Sono un vero Balilla [...]. Sono fiero della divisa che indosso, e quando sarò grande diventerò un prode soldato come vuole il nostro amato Duce».
} 
non citare il nome del Duce e la guerra che si combatte nei temi troppo distanti da questi argomenti». Riccardo Bruera, a cui è rivolta la critica, inserisce, infatti, l'esortazione di Mussolini ad essere operosi nel testo dal titolo «Anche gli animali lavorano: tra gli altri osserviamo quelli domestici, le formiche e le api».

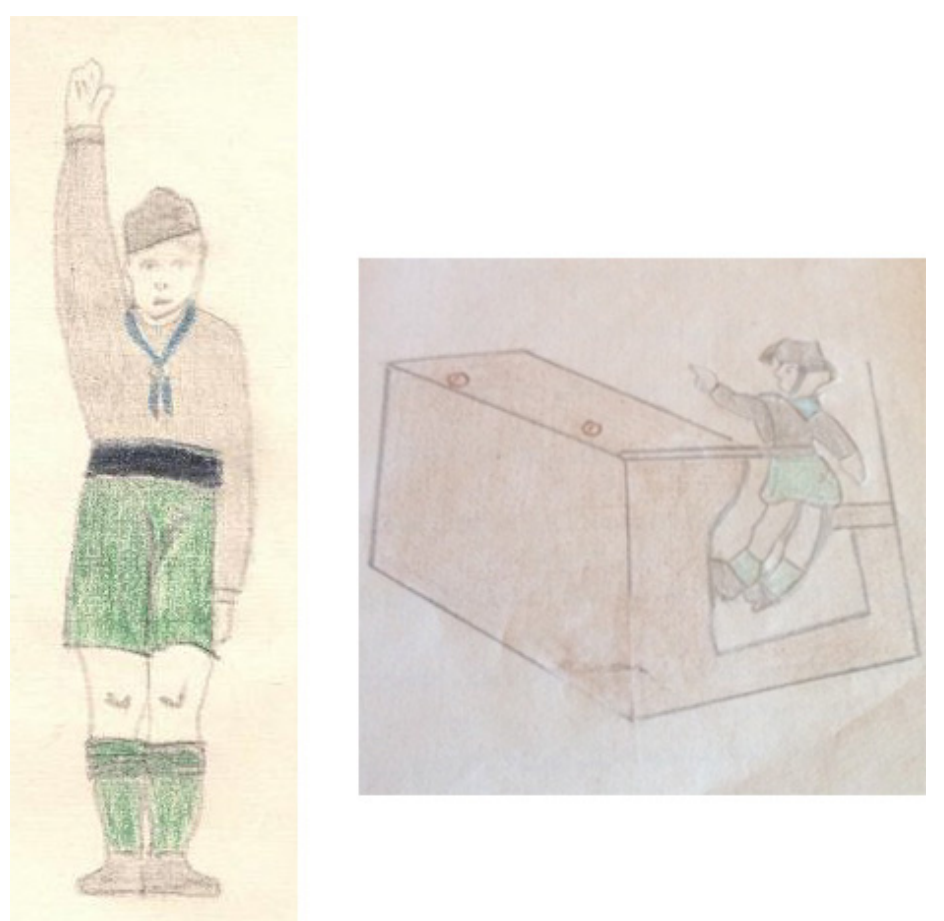

Figura 4. Esempi di identificazione nel modello del giovane Balilla.

I temi, seppur attinenti ad un argomento definito a priori dal maestro, consentono all'alunno di esprimere con maggiore libertà, rispetto al resoconto dei fatti di cronaca, idee ed emozioni. Costuiscono, quindi, un interessante e significativo elemento per cogliere la reale capacità di incidenza della propaganda ideologica del regime sugli animi e sulle coscienze delle giovani generazioni. Se è vero che la lettura di testi accomunati dallo stesso soggetto rivela un diverso livello di approfondimento e una maggiore o minore ricchezza di dettagli, è altrettanto vero che denota un'interiorizzazione degli ideali del fascismo e una granitica 
certezza nella realizzazione delle sue promesse. È sufficiente mettere a confronto il componimento di due alunni di quinta sulle difficoltà incontrate dalla popolazione nella stagione invernale in epoca di guerra: Gabriele Chiavassa colloca la strenua lotta agli sprechi nel più ampio quadro del conflitto bellico, descritto con un preciso e puntuale riferimento alla composizione degli opposti schieramenti, mentre Antonio Faccenda si focalizza esclusivamente sulla determinazione e forza morale degli italiani, di cui sottolinea lo spirito di sacrificio «veramente ammirevole», la «volontà tenace ed incrollabile» e la «semplicità sorprendente» nel sopportare disagi e privazioni; in entrambi, tuttavia, è presente, accanto alla convinzione di contribuire in forma «ideale» al combattimento con le economie nella vita quotidiana, la fiducia nella «immancabile vittoria», meritato tributo "ai popoli forti e sicuri del loro giusto diritto» e impegnati «a portare la luce della civiltà con quella della religione». Altrettanto significativa è la comparazione tra il tema di Sergio Bergamasco e quello del compagno di classe Guido Pugno relativi all'anniversario della marcia su Roma. Nei due scritti, oltre alla consueta celebrazione con tono retorico di elementi stereotipati (il ricordo della sfilata dei «bravi» squadristi nelle vie della capitale, l'idea della rivoluzione/insurrezione fascista, destinata a liberare la nazione dal pericolo del comunismo e ad avviarla verso «alti destini»), è evidente un diverso modo di vivere la ricorrenza: uno è attento alle manifestazioni esteriori dell'evento ( 'L'Italia è tutta imbandierata; dalle Alpi alla Sicilia è uno sventolio di bianco, rosso e verde. La nostra bella penisola appare come uno smagliante stivale tricolore»); l'altro è coinvolto in modo personale e non nasconde la sua partecipazione emotiva («Gli inni mi hanno fatto palpitare di gioia il mio cuore di Balilla, riempiendomi gli occhi di lacrime»).

Lo stesso tema della guerra è destinato a influenzare l'immaginario infantile: non a caso alcuni alunni indicano tra i giochi preferiti quello del soldato o la fabbricazione di aeroplani di carta con cui simulare scene di bombardamenti. Perfino coloro che dichiarano di prediligere attività ludiche senza alcuna attinenza con la realtà bellica non mancano di sottolineare l'utilità dei passatempi prescelti ai fini di un arruolamento, in prospettiva futura, nell'esercito: Chiavassa scrive, infatti, che la pratica del calcio, il gioco delle bocce e la passione per la bicicletta mettono in moto e, quindi, potenziano «un po' tutti i muscoli del corpo», contribuendo a 
formare una generazione di "giovanotti e uomini in buona salute, in pienezza di forze, atti al lavoro e fisicamente pronti ad essere buoni soldati per la difesa e la grandezza nostra Italia». ${ }^{28}$

\section{IL QUADERNO COME STRUMENTO DIDATTICO}

La lettura dei quaderni offre interessanti informazioni che spaziano dal programma di studi ai contenuti delle discipline; dagli arredi dell'aula ai sussidi e ai materiali didattici; dalle modalità d'insegnamento e di correzione degli errori ai criteri di valutazione del profitto a breve e medio termine; dalla verifica dei progressi nella carriera scolastica alla percezione soggettiva del livello di conoscenze e dei margini personali di miglioramento. Emerge anche un'idea di classe come comunità, basata sulla relazione alunni-insegnante, ma inserita nella più ampia rete delle scuole elementari del territorio come attestano le visite periodiche delle autorità scolastiche locali.

Nella lingua italiana è richiesto agli allievi di produrre, in media, un paio di testi scritti a settimana: uno sul diario; l'altro sul quaderno dei componimenti illustrati. Se il primo trae prevalentemente spunto, come ricordato, dalla cronaca, il secondo appartiene a una serie tematica che il maestro detta all'inizio di ogni mese con riferimento all'esperienza quotidiana dell'alunno ( $I$ Il mio ritratto fisico e morale»; «I miei giochi preferiti»; «Il mio banco») o ad argomenti di carattere storico («Come ti immagini la vita in un borgo medioevale») etico («Illustrate l'ammonimento "Siate buoni o figli" »; "Ovunque si lavora: in casa, a scuola, per le vie, nelle officine e nei campi») e scientifico (Le piante, le stagioni, la natura, gli animali), veicolati anche attraverso il ricorso ad esperimenti (Teoria dei vasi comunicanti e Legge di Archimede). Non manca la descrizione dei lavori manuali svolti in laboratorio (cartonaggio, falegnameria, verniciatura) né il resoconto delle uscite sul territorio, occasione per celebrare, in maniera diretta o indiretta, le scelte, non solo economiche, del regime: dalla sfilata, in divisa, alla lapide del municipio, che ricorda l'introduzione delle sanzioni contro l'Italia, alla visita al cimitero per rendere omaggio ai «ceppi dei martiri fascisti con corone di alloro», alla gita alla «Conigliera Nazionale» nei dintorni della città nell'intento

${ }^{28}$ Gabriele Chiavassa, I miei giochi preferiti: $i$ miei compagni di gioco, Temi, Classe IV, anno 19401941. 
di mostrare come, in linea con la politica autarchica promossa dal Duce, si possano produrre ottime pellicce anche con animali domestici.

Sono presenti anche alcuni accenni relativi alla luminosità, alle dimensioni e alle pareti dell'aula su cui fanno bella mostra il ritratto del Duce e quello del Re, le cartine geografiche e il crocifisso, reintrodotto nel 1923, anno in cui la riforma del ministro Giovanni Gentile aveva reso nuovamente obbligatorio l'insegnamento della religione nelle scuole elementari. ${ }^{29}$

Dalla lettura del tema «Io a scuola», assegnato in classe quarta, è possibile inoltre desumere notizie anche sulle altre discipline che completano il programma di studi del biennio elementare superiore: dall'aritmetica alla geometria; dalla grammatica alla storia; dalla geografia alle scienze naturali; dalla ginnastica, al canto, alla religione. Sono distribuite nei diversi giorni della settimana secondo un ordine fisso: il dettato il lunedì e il tema al sabato; l'educazione fisica il martedì e il giovedì; il disegno il giovedì e il sabato; l'aritmetica e l'attività laboratoriale il mercoledì. Rari e abbastanza generici sono i riferimenti ai compiti, definiti come «i doveri dello scolaro»: si tratta di un impegno quotidiano che prevede, «sotto la sorveglianza della mamma», lo svolgimento di esercizi, problemi o composizioni di lingua al pomeriggio e una breve ripetizione delle lezioni alla sera. Durante i periodi di vacanza l'impegno dello studio è meno gravoso e concentrato in alcuni giorni per lasciare spazio al riposo, alla santificazione delle feste, agli affetti e ai momenti di condivisione e di convivialità all'interno della sfera familiare: nel caso dello studente prossimo all'esame è il tempo ideale per un ripasso del programma dell'intero anno scolastico.

Meno agevole è individuare la frequenza con cui avvengono le esercitazioni di calligrafia: nei quaderni, infatti, non sempre compare la data in cui l'alunno ha svolto il compito assegnatogli dall'insegnante. Sulla base dei pochi fogli utilizzati, è ragionevole presumere che a questa disciplina

\footnotetext{
${ }_{29}$ Il $1^{\circ}$ ottobre 1923, Giovanni Gentile, ministro della Pubblica Istruzione, reinserisce la religione cattolica tra le materie previste nel ciclo elementare. Il provvedimento segna un'inversione di tendenza rispetto alle scelte operate dai governi dell'Italia liberale a partire dagli anni Settanta dell'Ottocento. Con i Patti lateranensi, firmati l'11 febbraio del 1929, l'obbligatorietà di tale disciplina viene estesa alle scuole secondarie. Per ulteriori approfondimenti Carla Ghizzoni, Educazione e scuola all'indomani della Grande Guerra. Il contributo de "La Civiltà Cattolica» (1918-1931) (Brescia: La Scuola, 1997), 175-207 e 275-299.
} 
vengano dedicate due o tre lezioni mensili: se in classe quarta figura una parte relativa all'acquisizione degli elementi base della "bella scrittura», in quinta si predilige la forma del dettato. Va d'altra parte tenuto presente che, in quegli anni, il calendario scolastico è spesso soggetto a interruzioni a causa delle già richiamate vicende belliche.

Attraverso la lettura dei quaderni è possibile ripercorrere alcuni momenti della vita scolastica: dall'inaugurazione delle lezioni con la celebrazione della funzione religiosa, alla consegna delle pagelle al termine di ogni trimestre, alla distribuzione dei premi in un'aula addobbata a festa alla presenza del clero e del provveditore agli studi. I testi degli alunni documentano, inoltre, le visite periodiche delle autorità scolastiche all'interno delle classi: se l'ispettore si limita a rivolgere parole di encomio, di incoraggiamento e di esortazione «a lavorare sempre di buona voglia per arricchire la nazione», 30 il direttore interroga sulle diverse materie, fa svolgere esercizi di analisi logica e grammaticale, semplici problemi ed equivalenze, esamina i quaderni e, se necessario, fornisce spiegazioni. È il caso, ad esempio, della descrizione del monumento cittadino a Vittorio Emanuele II con le considerazioni relative alle quattro aquile che, poste alla base, rappresentano le date delle guerre di indipendenza e della presa di Roma $(1848,1859,1866,1870)$.

Non mancano interessanti spunti relativi all'aspetto metodologico. Costante è l'uso della radio come strumento in grado di offrire informazioni sull'attualità (bollettini di guerra, discorsi del Duce) e di promuovere nella scolaresca la conoscenza della vita e delle opere di connazionali illustri che hanno contribuito a rendere degni di onore e di lode il nome e il genio italico: da San Benedetto, padre della civiltà occidentale, ad Antonio Pigafetta, primo navigatore a compiere insieme a Magellano il giro di circumnavigazione attorno al globo, a Giuseppe Verdi, compositore, nell'età del Risorgimento, di musiche «che erano squilli di tromba contro gli oppressori». ${ }^{31}$ La radio è protagonista anche di uno degli opuscoli della bibliotechina di classe dal titolo Notizie da casa: dall'ascolto dell'apparecchio il marinaio Cimarella, imbarcato su una nave da

\footnotetext{
${ }^{30}$ Riccardo Bruera, La visita dell'Ispettore, Diario, Classe IV, anno 1940-1941.

31 Gabriele Chiavassa, Giuseppe Verdi, Diario, classe IV, anno 1940-1941. Così l'alunno prosegue nel resoconto della trasmissione radiofonica dedicata al musicista emiliano: «A Milano, il suo nome stesso era diventato una bandiera: Viva V.E.R.D.I. Vuol significare: Viva=Evviva; V=Vittorio; E=Emanuele; $\mathrm{R}=\mathrm{Re}$; $\mathrm{D}=\mathrm{Di}$; I=Italia».
} 
guerra, apprende della nascita del primogenito Vittorio. Ciò non sorprende se si considera il notevole investimento del regime su questo mezzo di comunicazione di cui appare evidente, fin dall'inizio, l'utilità a fini non solo propagandistici, ma anche didattici. ${ }^{32}$

Altrettanto significativi sono gli elementi che consentono di individuare le modalità e i criteri di valutazione degli esercizi e dei testi scritti dagli allievi. Con l'ausilio di una matita o di un pastello colorato (rosso o azzurro) il maestro evidenzia gli errori ortografici, di sintassi e punteggiatura, sottolinea l'uso di verbi o termini inappropriati e aggiunge, se necessario, preposizioni o complementi alla frase. In alcuni casi provvede direttamente alla correzione, in altri si limita a segnalare gli sbagli e le inesattezze nella forma espositiva: sulla quantità qualche errore sfugge alla sua attenzione. La valutazione è espressa con il ricorso ad una scala che, articolata in una serie di gradi intermedi, spazia da scarso a lodevole: talvolta il giudizio, affiancato dal segno più o meno, contiene l'esortazione a migliorare la calligrafia, ad approfondire il contenuto, a suddividere i periodi lunghi, ad essere più accurati nei disegni, ad evitare le ripetizioni. Guido Pugno è poco ordinato, mentre Angelo Dotto non sempre si attiene, nello svolgimento del tema, a quanto richiesto dal titolo. ${ }^{33}$ L'alunno Chiavassa viene ripetutamente invitato a scrivere meglio ed è oggetto di rimprovero da parte dell'insegnante per l'abitudine ad incollare sul quaderno, negli elaborati di argomento religioso, illustrazioni a colori ritagliate da libri e riviste. Non mancano esempi in cui evidente è il tentativo di riprodurre, in maniera pedissequa, brani o periodi desunti da opere che inneggiano al fascismo. Emblematico è il commento «Dove l'hai copiato?» riferito alla cronaca dell'allievo Velatta sulla celebrazione dell'anniversario dei Patti Lateranensi.

\footnotetext{
32 Sull'introduzione della radio a scuola e sulla pubblicazione di specifiche riviste per insegnanti volte a favorirne un uso didattico efficace si vedano i contributi di Carla Ghizzoni «La radio per i ragazzi nei primi anni del fascismo (1925-1933), History of Education \& Children's Literature 2 (2018): 219-250, e "La radio a scuola durante il ventennio fascista in Italia», in Libro de Resúmenes del XIX Coloquio de Historia de la Educación «Imágenes, discursos y textos en historia de la educación» (El Escorial - Madrid, 19-22 de septiembre de 2017), ed. Sociedad Española de Historia de la Educación, Universidad Complutense de Madrid, Universidad de Alcalá, Universidad Nacional de Educación a Distancia (Alcalá de Henares: Fundación General de la Universidad de Alcalá, 2017), 257-260.

33 L'alunno è tenuto a produrre una nuova versione nel caso in cui il testo risulti completamente fuori tema. È quanto accade a Bruera, costretto a riscrivere il componimento sull'entrata di Gesù in Gerusalemme.
} 
L'esame dei quaderni consente, inoltre, di verificare il profitto degli alunni nel corso della carriera scolastica: di Pianea Luigi, Chiavassa Gabriele, Bruera Riccardo e Dotto Angelo disponiamo, infatti, della raccolta dei temi relativi alle ultime due classi della scuola primaria. La presenza dello stesso insegnante, in entrambi gli anni, costituisce una garanzia in merito all'uniformità dei criteri di valutazione. Dal confronto tra i voti del biennio si evince un generale miglioramento nella composizione in lingua italiana: lieve o moderato in alcuni casi, considerevole in altri. Se Pianea riesce a ridurre da 7 a 1 il numero dei giudizi sul livello della sufficienza, Bruera ottiene il massimo in tutte le prove scritte, superando, così, i buoni risultati raggiunti precedentemente: anche Dotto recupera, in quinta, le lacune linguistiche non attestandosi mai al di sotto del «buono». Costante nel rendimento è Chiavassa, destinato a confermarsi su valori di eccellenza. Di quest'ultimo è stato possibile reperire anche i «diari» e i quaderni di «bella scrittura» del ciclo elementare superiore: nei primi le valutazioni risultano sempre medio/alte; nei secondi compaiono, invece, alcune sufficienze che evidenziano la già criticata tendenza a non curare molto la calligrafia. ${ }^{34}$

È ragionevole supporre che il progresso riscontrato sia l'esito di una combinazione di fattori, legati alla buona volontà del singolo, al contesto di apprendimento e alle qualità umane e professionali del maestro. Se gli allievi manifestano spesso, nei loro scritti, il proposito di essere più studiosi, diligenti nell'esecuzione dei compiti e attenti alle lezioni, non mancano, però, di sottolineare il clima positivo all'interno della classe e i meriti dell'insegnante. I ragazzi, infatti, vanno volentieri a scuola, «officina della gioventù», dove "si formano e si educano le future forze della nazione»:35 frequentano volentieri le lezioni, considerate una preziosa occasione di apprendimento e un'utile esperienza in preparazione alla vita adulta. Addirittura entusiastico è il giudizio espresso da Sergio Bergamasco: «Le più belle ore della giornata — afferma lo scolaro di quarta- sono quelle che trascorro a scuola [...]. Amo la mia scuola come la mia seconda casa. Evviva la mia cara scuola»!36

\footnotetext{
${ }^{34}$ In classe quinta si registrano, comunque, dei miglioramenti: Chiavassa, infatti, ottiene per la prima volta, in questa disciplina, un paio di ottime valutazioni (lodevole + , lodevole -).

35 Antonio Faccenda, Lavoro intellettuale, lavoro meccanico, lavoro manuale, lavoro scolastico, Temi, Classe V, anno 1941-1942.

36 Sergio Bergamasco, Io a scuola, Temi, Classe IV, anno 1940-1941.
} 
Parole di apprezzamento sono rivolte all'insegnante, Aldo Portalupi, nei confronti del quale gli scolari nutrono sentimenti di stima, rispetto (spesso il sostantivo maestro è scritto con l'iniziale maiuscola) e sincera affezione: è definito con il ricorso ad aggettivi come «caro», "amorevole», «buono» e "paziente»; è presentato come un «fratello maggiore» sempre disponibile ad aiutare in caso di difficoltà e a ripetere i concetti poco chiari con l'ausilio di esempi. È una dedizione alla professione ancora più apprezzabile alla luce dell'elevata numerosità delle classi: l'unico dato certo di cui disponiamo è quello che, riferito alla quarta dell'anno scolastico 1940-41, attesta la presenza in aula di ben 36 allievi. ${ }^{37}$ Pochi sono i riferimenti al rapporto con gli altri alunni, chiaro indice della tendenza, alimentata dalla visione ideologica del fascismo, a promuovere relazioni di natura «verticale» e asimmetrica, basate sul principio dell'autorità e sull'adozione di una rigida disciplina. Solo Baratto e Bergamasco descrivono il proprio atteggiamento verso i compagni: il primo in termini ambivalenti con una oscillazione tra una condotta aggressiva ( Sono cattivo delle volte e bisticcio anche») e slanci di generosità («Se mi domandano qualche cosa la impresto loro volentieri»); il secondo in maniera soddisfacente («Sono gentile con i miei compagni e felice quando posso fare loro un piacere»).

Dalla lettura dei testi si possono desumere, infine, elementi di autovalutazione che denotano, negli allievi, una visione abbastanza oggettiva dei risultati raggiunti, delle potenzialità inespresse, dei margini di miglioramento e dei limiti caratteriali. Se Riccardo Bruera, pur dichiarandosi abbastanza soddisfatto del profitto, esprime il proposito di migliorare, Filiberto Guadagno non esita a definire «brutta» la sua pagella. Chiavassa è pienamente consapevole di essere uno dei migliori all'interno della propria classe ( Ho la grande soddisfazione di essere tra i più avanti nella classifica»), giudizio confermato da una valutazione «lodevole» e «buona» in quasi tutte le materie. Chiara è anche la sua percezione delle difficoltà nell'apprendimento logico-matematico: «Dei tre sufficienti —egli scrive- quello che più mi dispiacque ed impensierì

\footnotetext{
37 Non è stato possibile reperire il fascicolo personale dell'insegnante né presso l'archivio storico del comune di Torino né presso quello della scuola Parini. Non disponiamo, quindi, di nessuna informazione relativa alla sua vicenda umana e professionale.
} 
il babbo è quello di aritmetica. Ho promesso ogni sforzo per levarli tutti e tre specialmente quello di aritmetica». ${ }^{38}$

È un desiderio motivato dalla volontà di «accontentare» i genitori e il maestro e dall'esigenza di approfondire le conoscenze acquisite in prospettiva della prosecuzione nelle scuole medie. Lo studio è percepito, infatti, come strumento privilegiato attraverso cui raggiungere, oltre a un discreto grado di cultura, una "posizione» all'interno della società.

Gli alunni sono altrettanto sinceri nel riconoscere i propri difetti: Luigi Pianea ammette di non essere «tanto amante degli studi»; Luigi Baratto confessa di essere "svogliato" a scuola soprattutto nelle belle giornate in cui pensa ad «andare a giocare con i compagni»;39 Angelo Dotto afferma di essere «un po' troppo chiacchierone» e, quindi, spesso disattento diversamente da Sergio Bergamasco, preoccupato di «non dimenticare nulla» delle spiegazioni del maestro; Guido Pugno afferma di essere pigro e di entrare talvolta in aula dopo il suono della campanella perché «gli dispiace tanto al mattino lasciare il lettino caldo».

Sono pochi e semplici accenni che rivelano, tuttavia, le potenzialità di un interessante e ancora poco utilizzato strumento ai fini di una ricostruzione, in senso temporale, dei processi di valutazione scolastica nei periodi storici caratterizzati da eventi di natura eccezionale e in quelli "ordinari», in cui la vita quotidiana scolastica si svolge all'insegna della normalità nel contesto politico-culturale e socio-economico di appartenenza.

\section{Nota sobre la autora}

Maria Cristina Morandini è professore ordinario presso il Dipartimento di Filosofia e di Scienze dell'Educazione dell'Università degli Studi di Torino, dove insegna Storia della Pedagogia e Storia della scuola e delle istituzioni educative. È autrice di volumi, saggi e articoli sulla storia

\footnotetext{
38 Gabriele Chiavassa, La visita del Direttore, Diario, Classe IV, anno 1940-1941. Non è l'unico allievo a possedere delle lacune in tale disciplina: Bruera scrive di non riuscire quasi mai a risolvere i problemi, mentre Pugno definisce l'aritmetica la sua «bestia nera».

39 Il quaderno dei temi di Baratto è particolare rispetto agli altri per la presenza, al termine di ogni elaborato, della firma del padre come presa visione del voto assegnato dal maestro.
} 
della scuola italiana tra XIX e XX secolo con particolare riferimento agli ambiti dell'educazione femminile, dell'editoria scolastica, della manualistica e dei quaderni, delle memorie degli insegnanti, utili a ricostruire il percorso di formazione e l'attività didattica della classe magistrale. Negli ultimi anni si è dedicata anche allo studio della storia della pedagogia speciale, focalizzando l'attenzione, nello specifico, sull'educazione dei soggetti sordi, ciechi e rachitici.

Tra le principali pubblicazioni si segnalano Scuola e nazione. Maestri e istruzione popolare nella costruzione dello stato unitario (2004), La conquista della parola. L'educazione dei sordomuti a Torino tra Otto e Novecento (2010) e Punti e virgole, pesi e misure. Libri, maestri e scolari tra Otto e Novecento (2011).

\section{REFERENCIAS}

Andriola, Fabio. Luigi Rizzo. Roma: Ufficio storico della marina militare, 2000. Escolano, Agustín Benito (ed.). La cultura material de la escuola. Berlanga De Duero: CEINCE, 2007.

Ghizzoni, Carla. Educazione e scuola all'indomani della Grande Guerra. Il contributo de «La Civiltà Cattolica» (1918-1931). Brescia: La Scuola, 1997.

Ghizzoni, Carla. "La radio per i ragazzi nei primi anni del fascismo (1925-1933). History of Education \& Children's Literature 2 (2018): 219-250.

Ghizzoni, Carla. «La radio a scuola durante il ventennio fascista in Italia», in $L i$ bro de Resúmenes del XIX Coloquio de Historia de la Educación «Imágenes, discursos y textos en historia de la educación» (El Escorial - Madrid, 19-22 de septiembre de 2017), edito da Sociedad Española de Historia de la Educación, Universidad Complutense de Madrid, Universidad de Alcalá, UNED, 257-260. Alcalà de Henares: Fundación General de la Universidad de Alcalà, 2017.

Ghizzoni, Carla. «I quaderni scolastici: una fonte per la storia dell'educazione». Annali di Storia dell'Educazione e delle Istituzioni Scolastiche 13 (2006): 13-189. Lawn, Martin e Ian, Grosvenor, Materialities of Schooling: Design, Technology, Objects, Routines. Oxford: Symposium Books, 2005.

Lombardi, Franco V. I programmi per la scuola elementare dal 1860 al 1985. Brescia: La Scuola, 1987.

Loparco, Fabiana. "The MUSLI (Museo della Scuola e del Libro per l'Infanzia) of the Foundation Tancredi di Barolo in Turin. An institution at the forefront of the preservation and enhancement of educational and scholastic heritage». History of Education \& Children's Literature 1 (2013): 795-818. 
Meda, Juri. Mezzi di educazione di massa. Saggi di storia della cultura materiale della scuola tra XIX e XX secolo. Milano: Angeli, 2016.

Meda, Juri, y Ana Badanelli (a cura di). La historia de la cultura escolar en Italia y en España: balance y perspectivas. Macerata: EUM, 2013.

Meda, Juri, Davide Montino e Roberto Sani. School exercise books. A Complete Source for a History of the Approach to Schooling and Education in the $19^{\text {th }}$ and 20th Centuries. 2 vol. Macerata: Edizioni Polistampa, 2010.

Moegli, Pierre. Les industries éducatives. Paris: Presses Universitaires de France, 2010.

Montino, Davide. «Quaderni scolastici e costruzione dell'immaginario infantile». Annali di Storia dell'Educazione e delle Istituzioni Scolastiche 13 (2006): 167-189.

Montino, Davide. Bambini, penna e calamaio: esempi di scritture infantili e scolastiche in età contemporanea. Roma: Aracne, 2007.

Morandini, Maria Cristina. «La figura di don Bosco nella politica scolastica del regime fascista: uno sguardo ai manuali di pedagogia». In Percezione della figura di don Bosco all'esterno dell'Opera Salesiana, edito da Grazia Loparco e Stanislaw Zimniak, 121-137. Roma: Las, 2016.

Polenghi, Simonetta. "School subjects didactics in the history of education. Sources and methodology. Italian studies». History of Education \& Children's Literature 1 (2014): 635-648.

Sarfatti, Michele. Gli ebrei nell'Italia fascista. Vicende, identità, persecuzione. Torino: Einaudi, 2007. 